\title{
Rectal Metastases of Breast Carcinoma: A Case Report
}

\author{
Emilia Virginia Lima Curvelo Fontes, MD'; Nataliê Almeida Silva, MD'; Lister Arruda Modesto dos Santos, MD'; Vitorino Modesto dos
} Santos, MD, $\mathrm{PhD}^{2 *}$

'General Surgery Department, State Worker's Hospital, São Paulo-SP, Brazil

${ }^{2}$ Medicine Department, Armed Forces Hospital and Catholic University, Brasília-DF, Brazil

\begin{abstract}
The scarcely reported hematogenous rectal metastases from breast cancer are rare and the diagnosis is challenging. They may be recognized before, concomitantly with, or after the diagnosis of the primary site of breast cancer. Invasive lobular cancer is the histological type more frequently described, and most of the affected patients have a late diagnosis. Tardive recognition is associated with poor outcomes, despite the management options. Endoscopic and imaging evaluations, mainly magnetic resonance studies, are useful, but the anatomopathological findings are mandatory to confirm the diagnostic hypothesis. We describe a middleaged woman with advanced rectal metastases of unsuspected breast cancer found during the evaluation of manifestations due to intestinal implants. One must highlight long-term follow-up of breast cancers even if seeming in remission. The aim of this report is to enhance the suspicion index of primary health care workers.

Keywords: Breast cancer, Gastrointestinal tract, Invasive lobular cancer, Metastasis, Rectum

Cite this article as: Fontes EVLC, Silva NA, Santos LAM, Santos VM. Rectal metastases of breast carcinoma: a case report. Arch Iran Med. 2021;24(2):125-128. doi: 10.34172/aim.2021.19.
\end{abstract}

Received: October 6, 2020, Accepted: November 16, 2020, ePublished: February 1, 2021

\section{Introduction}

Metastasis to the gastrointestinal (GI) tract is uncommon, mainly if breast is the primary site; clinical manifestations are usually non-specific with symptoms related to local factors and extension of the bowel involvement. ${ }^{1-9}$ Breast cancer affects lymph nodes, bones, lungs, liver, brain, and soft tissues, but rectal spread is very rare..$^{2-9}$ Lobular carcinoma is a major subtype in GI implants with up to $18 \%$ of incidence. ${ }^{1,2,4-9}$ The period between the primary tumor diagnosis and evidence of implants is variable and reported to be up to 30 years. ${ }^{1,4,8,9}$ The diagnosis of breast cancer ocasionally occurs after manifestations of the rectal metastases. ${ }^{5,8}$ Worthy of note is a long time after the surgical treatment of breast cancer before the first manifestations and diagnosis of metastasis, increasing the diagnostic challenges as well as the unfavorable outcomes. ${ }^{1-3} \mathrm{Up}$ to $30 \%$ of breast cancers metastases occur after treatment, and $5 \%-10 \%$ are in stage IV. ${ }^{8}$ The authors believe that case reports may increase awareness of this very rare condition.

\section{Case Report}

A 43-year-old Brazilian female sought medical care because of obstipation, hematochezia, and weight loss $(8 \mathrm{~kg})$ in the last two months. There was also progressive nodule growth in the right breast. She denied smoking and alcohol abuse and had a history of partial left parathyroidectomy due to a pleomorphic adenoma. Physical examination showed body mass index (BMI): $33.3 \mathrm{~kg} / \mathrm{m}^{2}$ and breast asymmetry with fixed nodules of stone consistency bilaterally. Rectal examination revealed a vegetating lesion $1-2 \mathrm{~cm}$ from the anal verge, circumferential, with stenosis tendency, and bleeding. Mammography (Figure 1) showed thickening of the skin and the areola-papillary complex, accentuation of the breast parenchyma bilaterally and more evident on the right, in addition to lymph nodes with increased dimensions and density (BIRADS-5). Computed tomography (CT) revealed bilateral masses in the breasts and subcutaneous tissue, bilateral axillary lymph node enlargement, and concentric parietal thickening of the rectum associated with increased lymph nodes in the perirectal fat (Figure 2A and $\mathrm{B}$ ). This finding had a better definition on magnetic

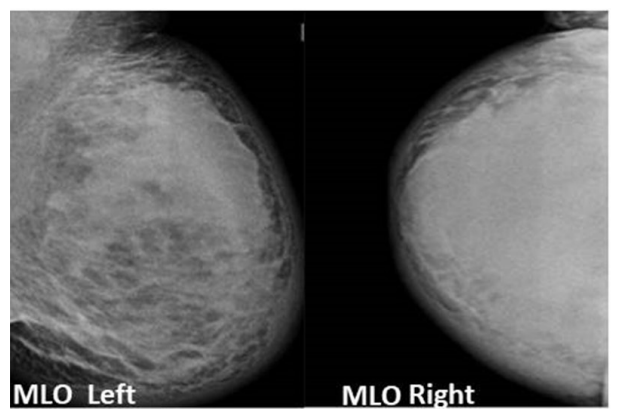

Figure 1. Mammography in the Medial-Lateral Oblique Positions Showing Bilateral Thickening of the Skin and the Areola Papillary Complex, Besides Coarsened Parenchyma More Evident on the Right, and Lymph Nodes with Increased Dimensions and Density. 
resonance imaging (MRI), showing an expansive lesion with irregular and circumferential contours in the middle and lower rectum, and anal canal, measuring $10.6 \mathrm{~cm}$ and at $0.7 \mathrm{~cm}$ from the anal border, besides lymph node enlargement in the mesorectum (Figure $2 \mathrm{~B}$ and $\mathrm{C}$ ). Bone scintigraphy showed abnormal accumulation of contrast in the right shoulder, mandible, L5-S1 vertebrae, and right ankle. Tumor markers showed high levels of CA 153: $427.3(\mathrm{VR} \leq 23.5) \mathrm{IU} / \mathrm{mL}, \mathrm{CEA}: 64(\mathrm{VR} \leq 10) \mathrm{ng} / \mathrm{mL}$, CA 125: $420.9(\mathrm{VR} \leq 35) \mathrm{IU} / \mathrm{mL}$, and CA 19.9: 38.7 (VR $\leq 35) \mathrm{IU} / \mathrm{mL}$. The patient underwent breast biopsy and proctological examination with biopsy. Histopathological study of the right breast sample was consistent with an invasive lobular carcinoma grade 2 nuclear, positive for estrogen receptor, negative for the progesterone receptor, cerb-B2, and e-cadherin, and with a proliferation index of $60 \%$. The study of rectal samples revealed a poorly differentiated carcinoma (Figure $3 \mathrm{~A}$ and $\mathrm{B}$ ) with an immunohistochemical pattern compatible with metastasis of the breast tumor (CK-7 and GATA3 were positive; CK-20, CD-X2, and estrogen receptor negative). Due to

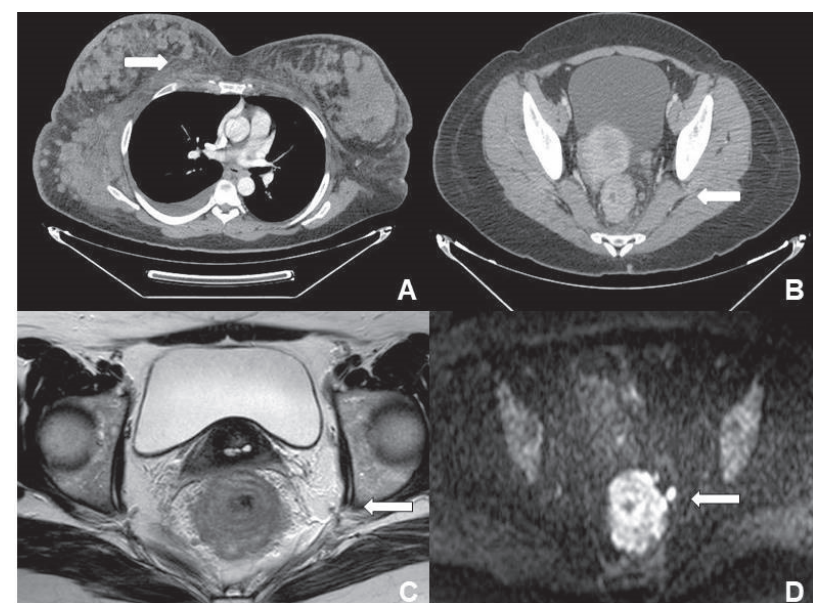

Figure 2. CT images of the chest and abdomen confirming bilateral subcutaneous and breast masses and bilateral axillary enlarged lymph nodes (A); and showing an increased volume of the perirectal fat (B). MRI axial images of the abdomen revealing accentuated concentric thickening of the rectal wall, associated with enlarged lymph nodes up to $0.8 \mathrm{~cm}$ in the perirectal fat $(C$ and $\mathrm{D})$.

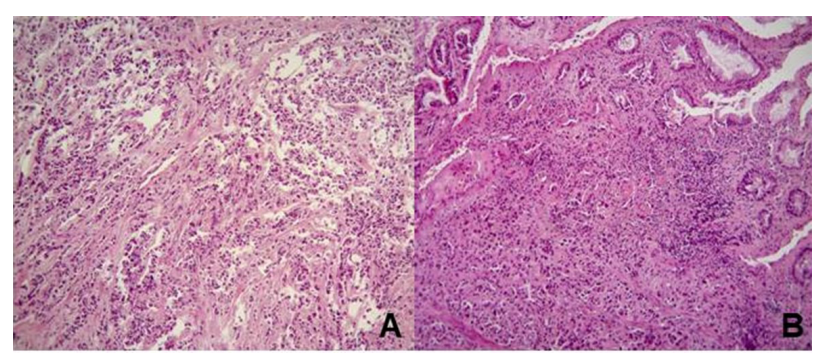

Figure 3. Photomicrographs of Biopsy Samples. (A) Invasive lobular carcinoma of the breast (Hematoxylin and Eosin, 10X). (B) Rectal metastasis of low-differentiated carcinoma with the primary site in the breast (Hematoxylin and Eosin, 10X). rectal stenosis, the management option was to perform a derivative colostomy. Her sudden death occurred while undergoing palliative radiotherapy for pain control.

\section{Discussion}

Female breast cancer is very common, affecting $12.5 \%$ of women during their lifetime., ${ }^{2,3,6-9}$ The risk of distant metastases from breast cancers is around 50\%. ${ }^{4}$ Breast tumors greater than $2 \mathrm{~cm}$ in diameter and metastatic lymph nodes are factors for distant implants, ${ }^{4}$ as observed in our patient with a neoplasm over $10 \mathrm{~cm}$ and osseous plus GI metastases. Implants of breast cancer to the GI tract are uncommon, especially to the rectal area. ${ }^{2-6,8,9}$ The middle-aged woman described here had an invasive lobular breast carcinoma that evolved unsuspectedly with very late detection during medical evaluation for accentuated weight loss and symptoms associated with advanced rectal metastases. The prevalence of these metastases in cases of invasive lobular adenocarcinoma of the breast is related to enhanced tropism to the GI tract, loss of cellcell adhesion molecules, and non-cohesiveness of the cells, and an elevated tendency for tissue infiltration. ${ }^{4}$ She had had parathyroidectomy to treat pleomorphic adenoma with hyperparathyroidism. Some studies strongly suggest that during the ten years before the diagnosis of primary hyperparathyroidism, the occurrence of breast, kidney, and skin cancers is increased. ${ }^{10}$ This association may be due to genetic factors, previous radiation, and hypercalcemia by overproduction of parathyroid hormone that would increase breast cancer incidence. ${ }^{11} \mathrm{CT}$ and MRI imaging, rectosigmoidoscopy, laboratory tests, and immunohistochemistry data established the diagnosis of breast lobular adenocarcinoma with rectal metastasis. Some reports emphasize the role of MRI soft tissue contrast to delineate the layers of the GI wall, allowing radiologic differentiation from primary rectal adenocarcinoma. ${ }^{3}$ The diagnosis of rectal metastasis and the discovery of breast cancer were concomitant. ${ }^{8}$ So, the discarded hypothesis of breast metastasis from rectal cancer by tumor markers and anatomopathological findings deserves a mention. Mammary implants from other solid malignancies are very uncommon $(0.3 \%-2 \%)$ and lung, nasopharynx, ovary, stomach, thyroid, and cervix are primary sites rarely reported, while rectal origin is exceptional. ${ }^{12}$ CT revealed bilateral breast and subcutaneous masses, and axillary lymphadenopathy. Even in advanced rectal cancers, breast metastases rarely occur, and unlike the present case, there is no bilateral involvement of the breast and axillary lymph nodes. ${ }^{12}$ Mammary metastasis is often unilateral, in the left upper outer quadrant, at a median age of 50 years, and adolescence, lactation, and pregnancy might enhance the incidence. ${ }^{12}$ The MRI images showed an expansive irregular and circumferential lesion involving the middle and lower rectum and anal canal, and enlarged lymph nodes in the mesorectum. The metastatic pathway 
in this setting could be hematogenous, starting by the entry of breast cancer cells into the axillary veins and following to venous vertebral plexus that transfers tumor emboli to pelvic organs through the posterior intercostal arteries. ${ }^{9}$ Endoscopic view of rectal implants include circumferential infiltration with thickening and rigidity of the rectal wall and narrowing of lumen, polypoid changes with a cobblestone pattern, and less frequently appearing as a solitary intraluminal mass. ${ }^{2-5,7-9}$ Besides the rectal carcinoma, the list of differential diagnoses can include inflammatory intestinal diseases, ischemic colitis, lymphoma, and other infiltrative malignancies. ${ }^{1,6-8}$ The survival time of women with GI metastases varies between 3 and 41 months, which is lower than 24-36 months observed in cases of metastatic breast cancer in other sites. ${ }^{2}$ About 53\% have some response to treatment but survival post-treatment is often poor. ${ }^{4}$ This rare entity has no consensual management and surgery is the option to treat cases of obstruction (colostomy, stenting, proctectomy, mesorectal excision), hematemesis, or perforation. Additional resources include aromatase inhibitor (letrozole, exemestane); chemotherapy (paclitaxel, docetaxel, anthracycline, cyclophosphamide, epirubicin, palbociclib); immunosuppression (everolimus); and palliative radiotherapy. ${ }^{2-6,8,9}$ Recurrences are usual both in the primary site and the site of distant breast cancer metastases. ${ }^{4}$ The current aging population with an increased number of cancer survivors should propitiate the occurrence of unusual presentations of breast cancer distant metastases. ${ }^{6}$ Some autopsy findings indicate that breast cancer metastases to the GI tract may be more frequent than clinically suspected and the true incidence is therefore not known. ${ }^{4}$ This issue merits a comment because of the current small number of postmortem studies, and a minimally invasive necropsy is an alternative to elucidate cancer causes of death. Researchers found the histopathological study of excised hernia sacs giving first clues for extra-abdominal cancer with GI metastasis, like the breast lobular adenocarcinoma. ${ }^{13}$ Exceedingly rarely, metastatic breast cancer gives origin to carcinomatous peritonitis. ${ }^{5}$ If one considers the analysis of hernia sacs allowing the investigation of peritoneal seeding or invasion, microscopic study of all abdominal wall hernia seems beneficial. ${ }^{13}$ MRI images contribute to distinguishing primary rectal carcinoma from GI metastasis. Concentric mural thickening with involvement of the submucosa and muscularis propria and sparing of the rectal mucosa are not usually found in primary rectal cancer. ${ }^{3}$ Immunohistochemical evaluation is useful to confirm the breast as the primary tumor site. The presence of gross cystic disease fluid protein (GCDFP-15) and of estrogen and progesterone receptors (ER and $\mathrm{PR}$ ) are strong evidence for breast origin. The GCDFP-15, ER, and $\mathrm{PR}$ are positive in metastasis and often negative in rectal cancer, while CK-7 positivity and absence of CK-
20 and CDX-2 commonly occur in breast cancer. ${ }^{7}$ We found very significant elevations in levels of CA-125 and CA 15-3 (12 and 18 times, respectively) above the upper normal values. CA-125 may be elevated in cases of metastatic breast carcinoma, because it can be expressed by cells of the mammary ducts. ${ }^{9}$ CA-125 detection occurs in up to $65 \%$ of patients with multiple metastases from breast cancer, and levels may have parallel responses to the tumor post-operative status. ${ }^{9}$ Further research is needed to confirm the practical utility of CA-125 in breast cancers. ${ }^{9}$

In conclusion, GI tract is a rare site for metastatic breast cancer, but a history of this tumor and fecal occult blood or bowel habit changes raise suspicion. Rectal metastases often mimic primary cancer. Prompt endoscopic and imaging studies reduce misdiagnosis and late diagnosis. Long-term follow-up is needed even in breast cancers seeming in remission.

\section{Authors' Contribution}

All the authors participate equally on the conception and design, collection and interpretation of data, literature search, and writing and review of the manuscript.

\section{Conflict of Interest Disclosures}

There were no potential conflicts of interest.

\section{Ethical Statement}

The authors followed the policy of the Committee on Publication Ethics (COPE) during writing this manuscript.

\section{References}

1. Blachman-Braun R, Felemovicius I, Barker K, Kehrberg E, Khan F. Widespread metastatic breast cancer to the bowel: an unexpected finding during colonoscopy. Oxf Med Case Reports. 2019;2019(2):omy133. doi: 10.1093/omcr/omy133.

2. Cherian N, Qureshi NA, Cairncross C, Solkar M. Invasive lobular breast carcinoma metastasising to the rectum. BMJ Case Rep. 2017;2017:bcr2016. doi: 10.1136/bcr-2016215656.

3. Lau LC, Wee B, Wang S, Thian YL. Metastatic breast cancer to the rectum: A case report with emphasis on MRI features. Medicine (Baltimore). 2017;96(17):e6739. doi: 10.1097/ MD.0000000000006739.

4. Ng CE, Wright L, Pieri A, Belhasan A, Fasih T. Rectal metastasis from breast cancer: A rare entity. Int J Surg Case Rep. 2015;13:103-5. doi: 10.1016/j.ijscr.2015.06.023.

5. Osaku T, Ogata H, Magoshi S, Kubota Y, Saito F, Kanazawa S, et al. Metastatic nonpalpable invasive lobular breast carcinoma presenting as rectal stenosis: a case report. J Med Case Rep. 2015;9:88. doi: 10.1186/s13256-015-0568-x.

6. Ruymbeke H, Harlet L, Stragier B, Steenkiste E, Ryckx M, Marolleau F. Anorectal metastasis from breast carcinoma: a case report and review of the literature. BMC Res Notes. 2018;11(1):268. doi: 10.1186/s13104-018-3356-z.

7. Schellenberg AE, Wood ML, Baniak N, Hayes P. Metastatic ductal carcinoma of the breast to colonic mucosa. BMJ Case Rep. 2018;2018:bcr2018224216. doi: 10.1136/bcr-2018224216.

8. Vande Berg P, Fonseca S, Al-Awa A, Rezai Monfared M, Delande S, Chamlou R, et al. A rectal metastasis of an unknown lobular breast carcinoma and its management. Acta 
Gastroenterol Belg. 2020;83(2):327-30.

9. Xue F, Liu ZL, Zhang Q, Kong XN, Liu WZ. Mesorectum localization as a special kind of rectal metastasis from breast cancer. World J Gastroenterol. 2015;21(14):4408-12. doi: 10.3748/wjg.v21.i14.4408.

10. Palmieri S, Roggero L, Cairoli E, Morelli V, Scillitani A, Chiodini I, et al. Occurrence of malignant neoplasia in patients with primary hyperparathyroidism. Eur J Intern Med. 2017;43:7782. doi: 10.1016/j.ejim.2017.06.001.
11. Michels KB, Xue F, Brandt L, Ekbom A. Hyperparathyroidism and subsequent incidence of breast cancer. Int J Cancer. 2004;110(3):449-51. doi: 10.1002/ijc.20155.

12. Ye Y, Wang Z, Xiao W, Han G. Breast metastases in advanced rectal cancer: a case report. Int J Clin Exp Pathol. 2020;13(5):1270-74.

13. Topal U, Gok M, Akyuz M, Oz AB, Arikan TB, Solak I, et al. Is histopathological evaluation of hernia sacs necessary? Arch Iran Med. 2020;23(6):403-8. doi: 10.34172/aim.2020.34. 